\title{
Biomechanical Simulation of the Fetal descent without Imposed Theoretical Trajectory
}

\author{
R. Buttin, F. Zara, B. Shariat, T. Redarce, G. Grangé
}

\begin{abstract}
The medical training concerning childbirth for young obstetricians involves performing real deliveries, under supervision. This medical procedure becomes more complicated when instrumented deliveries requiring the use of forceps or suction cups become necessary. For this reason, the use of a versatile, configurable childbirth simulator, taking into account different anatomical and pathological cases, would provide an important benefit in the training of obstetricians, and improve medical procedures. The production of this type of simulator should be generally based on a computerized birth simulation, enabling the computation of the reproductive organs of the parturient woman and fetal interactions as well as the calculation of efforts produced during the second stage of labor. However, apart from the commercially available robotized dummy simulators, very few virtual training tools using computational technologies have been developed. Unfortunately, all of these simulators approximate the expulsive forces of childbirth by imposing a pre-computed fetal trajectory. They have rather limited possibilities and would be unlikely to meet the versatility requirements described above. Besides, much research work has been carried out to simulate precisely birth-induced pelvic floor dysfunction and organ prolapse, with damage to levator ani muscles. All these simulators perform a detailed modeling of the levator ani muscles in interaction with a rigid fetal head, at high computational cost. However, they do not take into consideration many pelvic organs involved in the process of childbirth.
\end{abstract}

To reconcile the accuracy of results and computation time, we propose an approach that lies between the two classes of simulator described above in order to perform a realistic simulation of the descent of the fetus through the birth canal. In this paper we present the first stage of this work by focusing on the geometrical and biomechanical modeling of the main organs involved $(i . e$. the uterus, abdomen and pelvis of the parturient woman interacting with the fetus) based on the laws of continuum mechanics. At this stage, to verify the correctness of our hypothesis, we use finite element analysis, because of its reliability, precision and stability. In sum, our study improves work performed on childbirth simulators because:

- our childbirth model takes into account all the major organs involved in birth process, thus enabling many childbirth scenarios to be considered,

- fetal head is not treated as a rigid body and its motion is computed by taking into account realistic boundary conditions, $i$. $e$. we do not impose a pre-computed fetal trajectory,

- we take into account the cyclic uterine contractions as well as voluntary efforts produced by the muscles of the abdomen,

- a slight pressure is added inside the abdomen, representing the residual muscle tone.

R. Buttin, F. Zara and B. Shariat are with Université de Lyon, CNRS, Université Lyon 1, LIRIS, SAARA team, UMR5205, F-69622, France

R. Buttin and T. Redarce are with Université de Lyon, CNRS, INSA de Lyon, Laboratoire Ampère, UMR5005, F-69621, France

G. Grangé is with Maternité Port Royal, Groupe Hospitalier Cochin - Saint Vincent De Paul (Assistance Publique - Hôpitaux de Paris), F-75679, France Manuscript received ??; revised ??
The next stage of our work will concern the optimization of our numerical resolution approach to obtain interactive time simulation, enabling it to be coupled to our haptic device.

Index Terms-Medical training, childbirth simulator, biomechanical models, 3D simulation, continuum mechanics.

\section{INTRODUCTION}

Traditionally, medical training concerning childbirth for young obstetricians involves performing real deliveries, under supervision. However, this medical procedure becomes more complicated when instrumented deliveries requiring the use of forceps or suction cups become necessary. A survey by the AURORE (Association des Utilisateurs du Réseau Obstétricopédiatrique REgional - Association of the Users of the Regional Obstetric and Pediatric Network) network of the RhoneAlps region in France showed the number of complications related to the use of forceps or suction cups [1]. It appeared that out of 4,589 births, nearly 150 resulted in slight or serious lesions to the fetus. In addition, nearly $90 \%$ of obstetricians who participated in this survey approve of the use of childbirth simulation tools for the training of doctors. Moreover, in 20 years the number of Caesareans in France has doubled to reach $20 \%$ of births. This increase is due to the fact that young doctors do not dare undertake complex medical procedures. Thus, the use of these teaching tools could complement the training of obstetricians (generally considered to be too short) and improve medical procedures. The main objective is to render the obstetrician capable of opting for a forceps delivery, and therefore to decrease the number of Caesareans which may complicate future pregnancies.

Currently many simulators exist. In most common cases, simulators make medical training for instrumented delivery possible using a physical interface. Usually their interface is composed of several physical parts (an assembly of plastic pieces) which represent the anatomy of some of the organs concerned (generally the pelvis and the head of the fetus). In addition, a motorized articulated system drives these physical parts to simulate the interaction of the fetus with the organs of the parturient woman and the obstetrician. Thus this haptic device makes it possible to generate resistant forces that reproduce a sensation similar to that felt by the practitioner during delivery. Moreover, these simulators enable the practitioner to increase his experience due to the similarities between the anatomical representation given by plastic parts and reality. Some of these tools permit the simulation of instrumented deliveries using forceps [2]. For example, the Hopkins-designed 
birth simulator is oriented towards shoulder dystocia [3], [4] and the Noelle ${ }^{\mathrm{TM}}$ simulator marketed by Gaumard offers a complete robotized anthropomorphic system including fetal cardiac rhythm [5].

However these dummy tools are not very realistic and it would therefore be interesting to develop a more versatile and configurable tool, making it possible to take into consideration the various anatomical and morphological structures of the fetus and the parturient woman, corresponding to different pathological cases. Such a tool uses Virtual Reality (VR) techniques and is composed of two parts: a computational model part, simulating the birth process and a haptic interface. The implementation of the computerized simulation part could take place through the definition of a complete threedimensional anatomical representation of the maternal pelvis and the fetus. In the state of the art [6], there are two types of these simulators:

- Pelvic floor simulators designed to estimate pelvic floor dysfunction and organ prolapse or pelvic floor birthinduced injuries. Usually, these simulators perform a detailed biomechanical model of the levator ani muscles in interaction with a rigid fetal head during labor.

- Birth simulator based on a simplified biomechanical model of the female reproductive system and the fetus. Expulsive forces are approximated by imposing kinematic boundary conditions on the fetal head, imitating reality.

Our aim is to develop a versatile birth simulator that offers teaching scenarios at various levels of difficulty and which take into account some complex deliveries. However, we do not seek to obtain highly accurate simulation; rather a convincing simulation. Unfortunately, the "pelvic simulators" based on biomechanical models aim to create complex and accurate models, at the expense of a long computation time. Moreover, they do not take all the pelvic organs involved in the birth process into consideration. On the contrary, the "birth simulators" are simplistic and they do not take proper account of boundary conditions.

In order to fulfill our objectives, we propose an approach that lies between the two above-mentioned classes of simulator. It is based on a simplified, but realistic, biomechanical modeling of all the organs involved at the second stage of labor, allowing the calculation of stresses generated by the descent of the fetus, guided by the cyclic contractions of the uterine and abdominal muscles. The results of this calculation would then be entered into a haptic device in interaction with the trainee.

The first stage of our approach, is to ensure the degree of feasibility and realism of the computational part to obtain a realistic simulation of the second stage of labor. Consequently, in this paper, we propose a biomechanical model of the female genital system (uterus, abdomen, soft and bony pelvis) based on the laws of continuum mechanics. We obtain the calculation of the fetal trajectory during childbirth resulting from the interactions that occur between the fetus and the organs of the parturient woman. The Finite Element Method has been chosen as the numerical resolution technique to validate our approach due to its stability and precision. The second stage of this work will concern the optimization of our method to obtain real time performance, necessary for an interactive tool.

This paper is organized as follows. Section II presents a state of the art report on childbirth simulators and, more particularly, on the biomechanical models already developed in this context. In Section III, we present our model of the second stage of labor. For each organ, we present its functional anatomy and its geometrical and biomechanical models. In Section IV, we present the results obtained by analyzing the behavior of organs during the simulation and in Section V the input of our results into the BirthSIM haptic simulator [7], [8], [9]. Finally, Section VI presents our conclusion and the prospects for our work.

\section{STATE OF THE ART}

Training simulators are currently used in many areas such as aeronautics [10], and also in medicine as an instruction tool or as a medical support for surgery [11], [12], [13], [14], [15]. In the field of obstetrics and gynecology, a large survey of existing medical training simulators has been conducted by Gardner [16], [17] and one more specific to childbirth modeling by Li [6]. Moreover, in 2002 Letterie [18] explored the possible role of virtual reality for training in obstetrics and gynecology and concluded that Virtual Reality is a method that is potentially useful for this purpose.

The first Virtual Reality birth simulator was introduced by Boissonnat and Geiger in 1993 [19], [20]. This simulator makes it possible to adjust various geometric parameters such as pelvic organs or fetal morphology. However, this simulator is not equipped with a haptic device and is thus devoid of interaction with the user. It was not designed to train young obstetricians, but rather to establish a prognosis for the delivery by conducting a simulation of the fetal descent guided by a pre-computed imposed trajectory. Therefore the simulator did not take into account different delivery scenarios. In 2004, Kheddar [21] developed a simulator coupling a threedimensional biomechanical model of the fetus and pelvis to a three-axis haptic system representing the hands of the obstetrician. Similarly Obst [22] proposed a simulator based on biomechanical modeling of the birth process. Here again, in both these models the boundary conditions are not realistic and the simulation is based on an imposed trajectory, and therefore not able to take into account different pathological cases that have been identified.

On the other hand, many studies have been carried out to determine, as accurately as possible, fetal head deformation or injuries to pelvic floor muscles during the second stage of labor [6]. As previously stated, they do not take into account the entire birth process but they provide valuable information about the functional and biomechanical aspects of some of the organs involved in the birthing process. Here we present an overview of some of these studies. In 2001, Lapeer [23] presented a non-linear static finite element model of the deformation of a complete fetal skull, subjected to pressures exerted by the cervix during the first stage of labor. This model allows evaluation of the biomechanics of fetal head molding using a theoretical model of intra-uterine and 
head-to-cervix pressures. Moreover in 2004, Lapeer presented an augmented reality-based simulation of an obstetric forceps delivery [24]. This simulation is based on a virtual fetus model obtained from MR (Magnetic Resonance) and CT (Computed Tomography) images, and a real forceps delivery tracked with passive optical markers. The contact between the virtual skull and the forceps is then established and visible on the simulator. Then in 2005 , he tested the feasibility of a real-time mechanical contact model to describe the interaction between the forceps and the fetal head [25]. It was concluded that an explicit dynamic model to calculate the deformation of the main fetal skull bones only, or a quasi-static model to calculate the deformation of the fetal head in its entirety, can achieve real-time performance.

In addition, Martins [26], [27] studied the simulation of Levator ani (LA) muscles to observe pelvic floor dysfunctions. A neo-Hookean constitutive model has been used for the LA muscle. It is considered to be quasi-incompressible and isotropic with a single fiber direction. The material properties of pelvic floor muscles have been approximated by using data from heart tissues. A realistic model of the fetus, represented by tetrahedral elements, has been used. The fetus is almost undeformable with very high rigidity. By varying maternal and fetal head geometries, as well as some other parameters such as presentation, the maximum muscle stretch ratio has been computed. Recently, Li [28] also investigated the effect of mechanical anisotropy on the biomechanical response of the LA muscle during childbirth. He varied the relative rigidity between the fiber and the matrix components, whilst maintaining the same overall stress-strain response in the direction of the fiber. Thus, a fetal skull was passed through two pelvic floor models, which incorporated the LA muscle with different anisotropy ratios. Interactions between the LA muscle and the fetal skull were modeled during the second stage of labor using finite deformation elasticity and frictionless contact mechanics. Results showed a substantial decrease in the magnitude of the force required for delivery as the fiber anisotropy was increased.

Furthermore, Mizrahi and Karni [29] have presented a mechanical model of the uterus using a kinematic approach. The expression of the strain gradients and strain compatibility for the middle surface of the shell in curvilinear-oblique coordinate networks are produced. As boundary conditions, they considered that the displacements of the cervix are zero in a single contraction and remain constant during the second stage of labor when the cervix is fully dilated. Moreover, they assumed the volume bounded by the organ to be constant during the deformation, due to the incompressibility of the inter-uterine fluid. They also presented [30] a study to improve the anisotropic behavior of the uterine muscle.

Contrary to these "precision driven" approaches, our work aims to represent realistic material properties and boundary conditions for all the organs involved in the second stage of labor [31]. Moreover, our aim is to maintain a balance between accuracy and computational complexity. For this reason, our biomechanical and geometric models have been simplified to reduce the overall computation cost.

\section{OUR MOdEL OF THE SECOND STAGE OF LABOR}

Delivery is a complex physiological phenomenon involving many organs. It must be remembered that the embryo develops during gestation in the uterus. Then, during the three stages of labor, the uterine contractions combine with the forces of the abdomen and diaphragm to expel the fetus. The second stage of labor starts at full dilatation of the cervix until the birth of the baby. During its descent, the fetus will cross the pelvic inlet (superior pelvic strait) and the pelvic outlet (inferior pelvic strait). Consequently, the head of the fetus which is the widest part, will deform the pelvic floor muscles to extricate itself from the utero-vaginal canal. Note that for our simulation, we consider the most common head presentation, e.g. the occiput anterior $(\mathrm{OA})$ presentation.

\section{A. Computational Model}

In this section we briefly present the computational methods used in our work. These concern the study of constitutive equations that connect applied stresses to body deformations, as well as the biomechanical parameters and assumptions about the organs involved.

1) Constitutive Equation: We have used two constitutive equations for the simulation of the organs: Hooke's and NeoHooke's laws. Hooke's law allows the modeling of linear elastic behavior. The elasticity means that the state of the deformation of the object depends only on the present state of the stress. Thus, an elastic material that is deformed under the action of certain forces returns to its original state once the forces disappear, and the absorbed energy is restored. To this we add linearity, that is to say that the forces are proportional to strain, and isotropy, which means that the properties of the object are the same in all directions. For homogenous and isotropic materials, Hooke's constitutive law is thus defined by:

$$
\sigma=D \cdot \epsilon
$$

with $\sigma$ being the stress tensor, and $\epsilon$ the strain tensor. The tensor $D$ is defined by

$$
[D]=\left[\begin{array}{cccccc}
\lambda+2 \mu & \lambda & \lambda & 0 & 0 & 0 \\
\lambda & \lambda+2 \mu & \lambda & 0 & 0 & 0 \\
\lambda & \lambda & \lambda+2 \mu & 0 & 0 & 0 \\
0 & 0 & 0 & \mu & 0 & 0 \\
0 & 0 & 0 & 0 & \mu & 0 \\
0 & 0 & 0 & 0 & 0 & \mu
\end{array}\right]
$$

with $\lambda$ being the Lame's first parameter and $\mu$ the shear modulus defined by:

$$
\lambda=\frac{E \cdot \nu}{(1+\nu) \cdot(1-2 \nu)}, \mu=\frac{E}{2(1+\nu)},
$$

with $E$ being the Young's modulus and $\nu$ the Poisson ratio of the material.

For the modeling of an incompressible hyper-elastic behavior, the Mooney-Rivlin model fits better the experimental data than Neo-Hooke's law, but requires additional empirical constant. In our case, the high precision is not our goal and because of the difficulties inherent in obtaining in vivo data, 
the introduction of an additional parameter will probably not improve the results. For this reason, we choose Neo-Hooke's law, for the modeling of an incompressible hyper-elastic behavior, which is characterized by a function of strain energy $W$, depending only on the current state of the deformation with $\sigma=\frac{\partial W}{\partial \epsilon}$. The strain energy is defined by:

$$
W=C_{10}\left(\overline{I_{1}}-3\right),
$$

with $C_{10}=\frac{1}{2} G$ and $G=\frac{E}{2(1+\nu)}$ the shear modulus and $\overline{I_{1}}$ first invariant of the left Cauchy-Green dilation tensor defined by $B=F \cdot F^{T}$ where $F$ is the gradient tensor of the transformation.

2) Mechanical Properties: The main difficulty in using these constitutive laws remains the choice of the values for these biomechanical parameters $(E, \nu$ and $C 10)$. Indeed, the exact values of mechanical properties are extremely difficult to determine and may vary by a factor of one thousand depending on the protocol used to determine them. Moreover, the values obtained in vitro are usually not appropriate, and it is often difficult to perform the experiments in vivo.

For example, Mazza et al. [32] present a study performed with an aspiration device to characterize the mechanical properties of human uterine cervices in vivo. The average values of the stiffness parameter vary from 0.095 to $0.24 \mathrm{bar} / \mathrm{mm}$, for experiments on eight patients, aged from 47 to 69 years and having had 1 to 4 births. However, it is difficult to use these values obtained in non-pregnant women because of the significant changes in mechanical properties during pregnancy [33]. For this reason, Bauer et al. [34] present another study performed with the same device but on pregnant women (between 21 and 36 weeks' gestation). They obtained stiffness values between 0.013 and $0.068 \mathrm{bar} / \mathrm{mm}, i$. e. nonpregnant tissue was significantly stiffer than pregnant tissue in both tension and compression.

In our work the mechanical parameters $C 10, \nu$ and $E$ have been set at the values found in [33] and [35].

3) Incompressibility Assumption for Organs: As the human body is composed of almost $90 \%$ water (incompressible material), its density is just below $1,000 \mathrm{~kg} / \mathrm{m} 3$ (with dense parts being mainly in the muscular areas) and the incompressibility assumption could be made for almost all modeled organs. Let us consider the equation for the conservation of the mass of a system:

$$
\frac{d \rho}{d t}+\rho \operatorname{div}(U)=0
$$

with $\rho$ being the density and $U$ the displacement. The incompressibility assumption is that $\frac{d \rho}{d t}=0$. Thus, we have $\rho=0$ or $\operatorname{div}(U)=0$. As the density of organs cannot be zero, we impose the condition $\operatorname{div}(U)=0$ to the displacement of organs.

\section{B. Selection of Organs to Model}

Many organs are involved during the second stage of labor. To simplify our model, we considered only the essential components, that is to say the uterus, abdomen and soft and bony pelvis, as well as the fetus. We have not modeled the placenta, the rectum and the bladder.
Indeed, the placenta is a relatively thin body which is located inside the uterine pocket. Mechanically, this body causes only a partial increase in the thickness of the uterine wall. The placenta is released a few minutes after the fetal exit, during the third stage of labor known as "delivery of the placenta". Since we focused only on the second stage of labor, we have not integrated the placenta into our model. However, modeling it will result in a higher computation time due to the treatment of contacts, without a significant impact on the simulation.

The bladder is rather imposing because it may contain about $350 \mathrm{ml}$ of liquid. However, at the beginning of labor it is emptied, significantly reducing its size and limiting its effect on the simulation of organ motion. Therefore, this body has not been integrated into our model. In the same way, the rectum does not have an important role during labor, and modeling it does not represent a real contribution to the accuracy of the results. Consequently, we have not incorporated the rectum in our model.

In the following sections, we will present the modeling aspects for each organ (pelvis, fetus, abdomen, uterus) and the forces (uterine contractions and expulsion forces) involved in our simulation. Note that the geometry of the various organs has been extracted from MRI data for soft tissues and CTscan data for the bony parts of pregnant women. This data was provided by the Saint Vincent de Paul Hospital (AP-HP) in Paris. They were then processed to obtain the mesh of the organs.

\section{Pelvis Model}

1) Functional Anatomy: The pelvis is composed of a bony section and a muscular section as illustrated in Fig. 1. The bony section is composed of three bones: the left and right iliac wings and sacro-lumbar spine. These bones are connected to each other by the muscular section of the pelvis, by a set of ligaments. This network of perineal muscles in the pelvis, located at the pelvic outlet, is commonly called "pelvic floor".

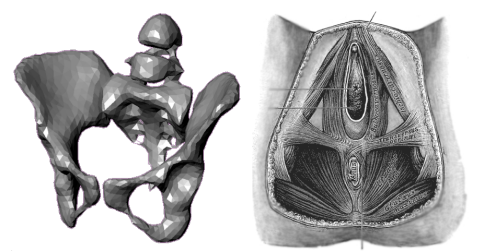

Fig. 1. Bony (left) and muscular (right) sections of the pelvis.

The pelvis is a key element in delivery with a resistive role for the pelvic floor which surrounds the lower part of the uterus and the vaginal area. From a mechanical point of view, the muscular section of the pelvis behaves in an elastic manner and can undergo large deformations.

The bony pelvis also plays an important role by guiding the fetal head into the birth canal. The pelvis then performs a nutational movement composed of two dependent rotations: a forward tilting of the sacrum when the fetal head is introduced into the vaginal canal, and an abduction of the iliac wings 
resulting in a decrease in the promonto-retro-pubic diameter as well as an increase of the sub-sacra-pubic diameter. The purpose of this variation in diameter is to facilitate the fetal descent, allowing the birth canal to enlarge. From a mechanical point of view, the bony pelvis behaves in an elastic manner with small deformations and small displacements.

2) Geometrical Model: We have seen that the pelvis is composed of two sections. For ease of calculation, the muscular section has been incorporated into the abdomen of the parturient woman. For the bony section, the mesh obtained directly from the CT-scan data is very noisy and complex $(1,752,152$ nodes $)$. So, we smoothed it [36] and we obtained a mesh with 18,300 nodes. To reduce the computational time of the simulation, we simplified it to preserve only its functional features (ischial spines, tip of the coccyx and pubic area) and removed the sharp edges. To do this, we first made a very coarse mesh which is based on bounding boxes of different connected parts of the pelvis, and finally we obtained a mesh with 1,750 nodes (see Fig. 2).
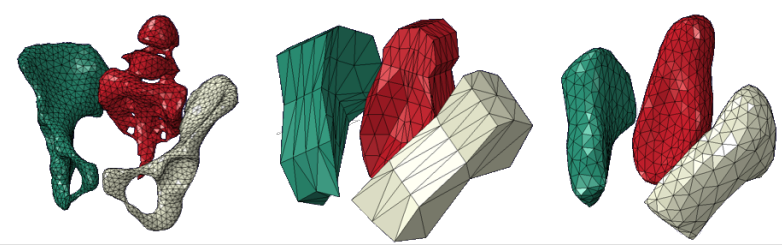

Fig. 2. Simplification of the bony pelvis mesh: (from left to right) smoothed mesh (18,300 nodes), bounding boxes, final mesh (1,750 nodes).

3) Biomechanical Model: We consider the iliac wings of the bony pelvis to be stationary and undeformable, the upper sacro-lumbar spine to be fixed and we have only allowed a rocking motion at the lower level. Fig. 3 shows these boundary conditions.

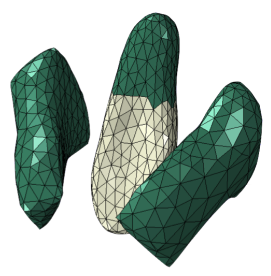

Fig. 3. Boundary conditions of the bony pelvis: in green the fixed parts.

For the mechanical behavior, Fung [33] recalls experiments performed by Yamada [37] suggesting that Hooke's law is applicable to bones for a limited range of strains. For this reason, we used the Hooke's law model for the bony pelvis that enables small deformations. For the Young modulus of the bone, Dufour and Pillu [35] presented an average value from 15 to $19 \mathrm{MPa}$. This average value includes the trabecular and cortical parts of the bone. Cortical bone being much denser than trabecular bone (the spongy part of the bone), we chose a Young modulus $E=23 \mathrm{MPa}$ to focus on the cortical bone. The Poisson ratio for bone is between 0.2 and 0.3 [38], so we chose $\nu=0.3$ for the Poisson ratio of the bony pelvis. Moreover, we estimated the density value at $1,000 \mathrm{~kg} / \mathrm{m} 3$, close to that of water.

\section{Fetus Model}

1) Functional Anatomy: We will now consider the fetus. From a mechanical point of view, it should be viewed as a very small human. It is, therefore, composed of different organs (materials) each with their own laws of behavior, mechanical properties and density. From an anatomical point of view, the proportions between the different parts of the body of the fetus are not the same as for an adult or a child. Indeed, the fetal head is highly developed compared to the rest of its body. Moreover, the fetal skull is composed of several bones with a global plastic behavior [39], [23].

2) Geometrical Model: From a geometrical point of view, the fetus assumes a tuck position within the uterus to reduce space. When in his position, the approximate measurements for height are $30 \mathrm{~cm}$ and width $12 \mathrm{~cm}$, with a weight of $3.5 \mathrm{~kg}$ [39]. A first simplification of the geometrical model of the fetus has been performed to smooth the mesh and eliminate complex elements as illustrated in Fig. 4. Consequently, the number of mesh nodes is reduced from 21,500 to 2,800 .
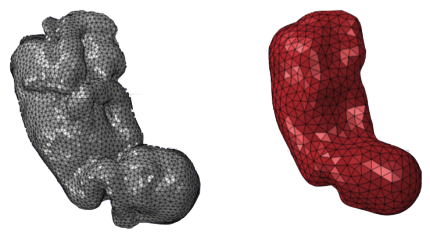

Fig. 4. Simplification of the geometrical model of the fetus: 21,250 nodes before (left) - 2,800 nodes afterwards (right).

3) Biomechanical Model: From the point of view of complexity, it is not possible to model all the different organs of the fetus. Therefore, we consider the fetus to be composed of three parts: the skull, the body and the skin tissue (cf. Fig. 5). Moreover, the skull and the body are included in the skin tissue (with several nodes in common), so we do not have to manage contact between these three parts.

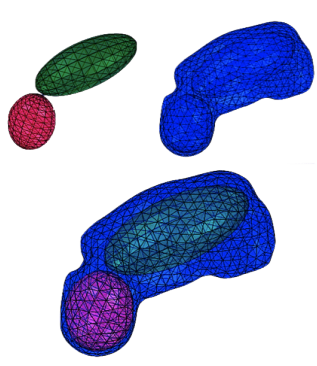

Fig. 5. The model fetus is composed of three parts: skull (red), body (green) and skin tissue (blue).

The skull is considered to be a deformable object as it undergoes significant deformation during delivery. The body is regarded as an object that is slightly deformable to allow the back of the fetus to move freely and to simulate the various joints. The skin tissue is considered more elastic than the body and the skull, with a lower elasticity modulus. It should be noted that the skin tissue is the only compressible organ in our model, to reduce the repulsion forces involved by contacts between uterus and fetus. 
The three parts of the fetus were modeled as Neo-Hookean materials with $C 10=130 \mathrm{kPa}$ for the skin tissue, $C 10=$ $75 \mathrm{kPa}$ for the skull and $C 10=70 \mathrm{kPa}$ for the body. Then, assuming that a fetus has a lower muscular density than an adult, we have considered the average fetal density to be slightly lower than $1,000 \mathrm{~kg} / \mathrm{m} 3$ : we use a density of $400 \mathrm{~kg} / \mathrm{m} 3$ for the skin tissue, and similar densities of $950 \mathrm{~kg} / \mathrm{m} 3$ for both the skull and the body.

This model enables the fetus to be simplified to a large degree, while preserving articulation of the skull, induced by the deformation of the skin tissue ( $c f$. Fig. 6).
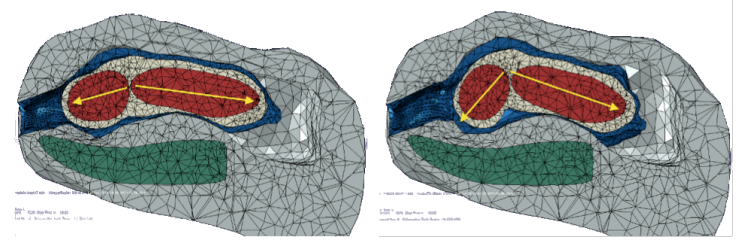

Fig. 6. Articulation of the fetal head made possible by deformation of skin tissue.

\section{E. Abdomen Model}

1) Functional Anatomy: We should now consider the abdomen which is made up of a large number of organs (bladder, rectum, spinal column, ribs, liver, etc.). During pregnancy, its volume increases significantly, and decreases during labor. Consequently, the organs of the abdomen are displaced and deformed around the fetus during pregnancy, and during the second stage of labor the descent of the fetus frees the occupied volume allowing the organs to resume their original position. This phenomenon is due to an internal pressure that enables the cohesion of the organs to be maintained.

2) Geometrical Model: Due to the computation time involved, it is not possible to model all the organs of the abdomen. For this reason, we will consider the abdomen as a single organ. The contour of the abdomen has been extracted from the MRI data and modeled with tetrahedral elements. Then the volumes of internal organs (pelvis, uterus and fetus) are subtracted to obtain a mesh with 38,863 nodes. Finally, we used the same simplification as for the fetus and obtained a mesh with 3,268 nodes ( $c f$. Fig. 7).

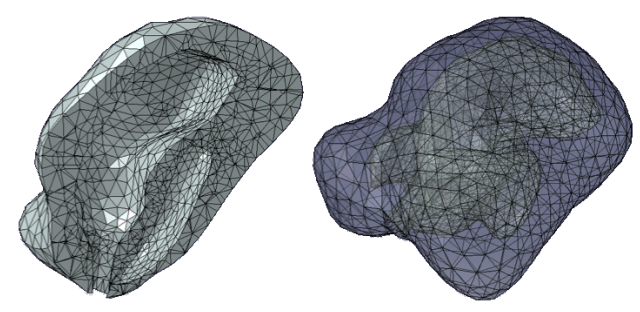

Fig. 7. Mesh of the abdomen of the parturient woman (right) with the sagittal view (left).

3) Biomechanical Model: As previously explained, we incorporated the pelvic floor into the abdomen model. Thus, we have provided mechanical behavior for the abdomen close to that for the muscular pelvic tissues, i.e. elastic and compressible. These properties enable the repositioning of the constituent elements during the descent of the fetus. Finally, the abdomen was modeled as a hyper-elastic material using the Neo-Hooke constitutive law with a density of $2,500 \mathrm{~kg} / \mathrm{m} 3$ and $C 10=5 \mathrm{kPa}$.

For the boundary conditions, we considered the back of the parturient woman (i.e. the rear part of the abdomen) to be rigid, to take into account the fact that the parturient woman is seated in an obstetric chair. However, we cannot impose zero displacement on all the abdomen contours. Indeed, if we fixed the lower part of the abdomen, the enlargement of the vagina would become impossible, preventing the fetus expulsion. This problem is resolved by allowing only lateral displacement of lower part of the abdomen around the vaginal area ( $c f$. Fig. 8).

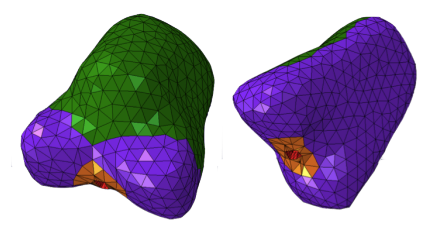

Fig. 8. Boundary conditions for the abdomen model: (purple) the fixed parts, (orange) the vulva can be stretched, (red) vaginal area vertically blocked, (green) all displacements permitted.

Moreover, to retain cohesion between the organs (uterus, pelvis and fetus) inside the abdomen, we added a slight pressure, representing the residual muscle tone. Fig. 9 illustrates this pressure effect in $2 \mathrm{D}$.
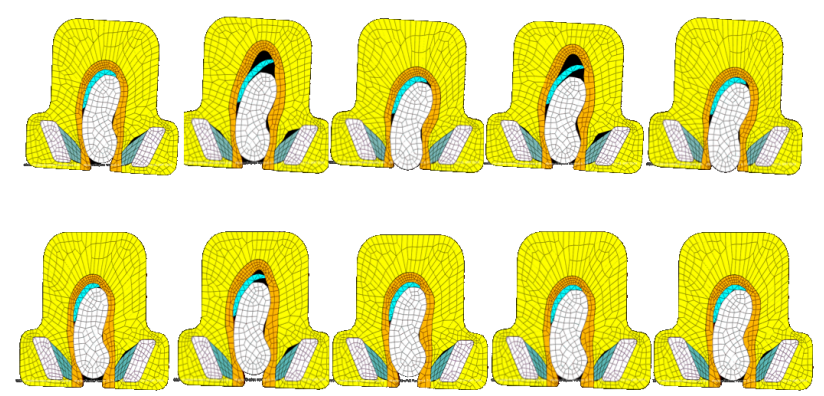

Fig. 9. Behavior of organs without/with internal pressure (top/bottom).

For the management of contacts, as the bony pelvis is included in the abdomen with several nodes in common, we do not have to detect any collision between these two organs.

\section{F. Uterus Model}

1) Functional Anatomy: The uterus is a thin closed shell membrane in which the fetus develops during pregnancy. Its average size for a non-pregnant woman is approximately $65 \mathrm{~mm}$ long, $45 \mathrm{~mm}$ wide and $30 \mathrm{~mm}$ thick. Its interior volume can increase almost 170 times compared to its initial volume during gestation [40]. This constant high tension applied to its muscular tissues during the nine months of pregnancy causes significant change to its mechanical properties, making them difficult to evaluate.

During labor, the uterus is the most important organ in the pelvic system since it supports all the efforts applied by other organs. Moreover during the second stage of labor, the uterus 
exerts a pressure on the fetus, pushing it into the birth canal. The inner walls of the uterus are flattened against the body of the fetus, decreasing the uterine volume throughout the descent, until the muscular membrane forms only a small clot in the perineal area. Finally, its height is approximately one third of its original height. Consequently, from a mechanical point of view, the uterus has an elastic behavior with high deformation.

2) Geometrical Model: To simplify matters, we modeled the uterus, cervix and vagina as a single unit. Fig. 10 shows its geometrical model. On the left, we can see the mesh directly obtained from medical data $(42,811$ nodes $)$. It does not contain the vaginal canal. On the right, we can see the mesh obtained after smoothing (2,348 nodes) with the vaginal canal reconstructed manually according to the anatomy [39], [41] and by considering a complete cervical dilation.
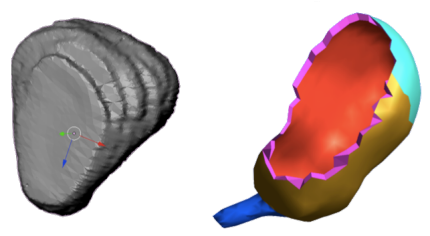

Fig. 10. Geometrical model of the uterus: (left) model directly obtained from medical data, (right) final mesh after smoothing and reconstruction of the vaginal canal.

3) Biomechanical Model: The works of Mizrahi showed that the behavior of uterine muscles changed during the childbirth, with an isotropic behavior in the early stages of childbirth and an anisotropic one with the progress of labor [30]. To simplify our model, we consider an anisotropic behavior for the uterine membrane. So the uterus has been modeled as a Neo-Hookean hyper-elastic material with a density of $950 \mathrm{~kg} / \mathrm{m} 3$ and $C 10=30 \mathrm{kPa}$. As boundary conditions, the displacements of the vaginal canal are limited in the transverse plane to allow the opening and closing of the vaginal canal, avoiding descent of the organs.

The contacts between the uterus and the fetus are considered to be frictionless. Indeed, when the labor phase begins, the amniotic fluid drains out of the uterus, but the internal walls are nonetheless fairly well lubricated. Moreover, the contacts between the uterus and the abdomen are also considered to be frictionless because of the viscous contact between all the organs within the abdomen.

\section{G. Uterine Contractions and Expulsion Forces}

1) Functional Anatomy: We have seen that the uterus is a muscular pouch. Instead of modeling the muscle behavior, we model its consequences, i.e. uterine contractions (UC). These uterine contractions are involuntary. They occur 3 or 4 times every ten minutes (one period). The average duration of a contraction is 90 seconds. The amplitude of the contraction varies between "base tonus" (pressure prevailing in the uterus caused by strong deformation) and the intensity of the UC. The true intensity is the difference between these two amplitudes. It corresponds to the effective thrust forces of the uterine contractions during delivery ( $c f$. Fig. 11) [39]. However, this thrust is insufficient to allow the effect of the pelvic muscles to be deleted, and delivery of the fetus. Therefore, during the second stage of labor, the parturient woman must voluntarily produce a series of significant abdominal thrusts synchronized with the uterine contractions. Indeed, even if these forces (called expulsion forces) are about 4 times higher, it is essential they are added to the UC to exceed the threshold necessary to overcome pelvic floor resistance and expel the fetus ( $c f$. Fig. 12).

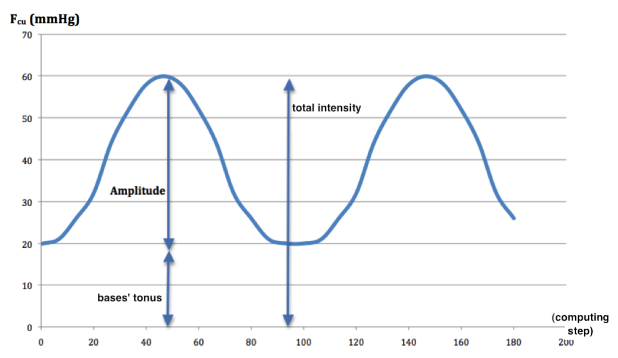

Fig. 11. Uterine contraction force $(\mathrm{mmHg})$ versus time.

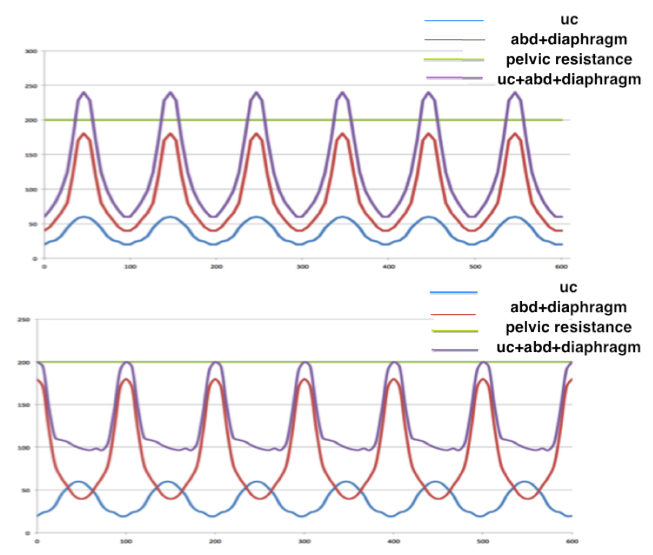

Fig. 12. Evolution of different uterine forces: synchronized forces (top) and unsynchronized forces (bottom). The green line is the delivery threshold.

These expulsion forces are caused by the contraction of the abdominal muscles and the diaphragm. The abdominal muscles are located in the lower abdomen, but they are lifted because of the presence of the fetus. Consequently, they encompass the uterine surface and exert uniform pressure on the top of the uterus. The diaphragm also pushes the fetus toward the vaginal canal. Finally the descent of the fetus is caused by the combination of the uterine forces and expulsion forces (abdominal and diaphragm forces) applied on the uterus, which shrink the uterine walls causing a force that expels the fetus into the vaginal canal.

2) Biomechanical Model: Mimicking reality [39], the uterine contractions and the expulsion forces are modeled as two periodic force fields on the inner and outer surface of the uterus, with 12 periods for a labor period of 30 to 40 minutes. Fig. 13 is an illustration: in gray, the part of the uterus on which the UCs are applied; in green, the part of the uterus on which the UCs, abdominal and diaphragm forces are applied. 

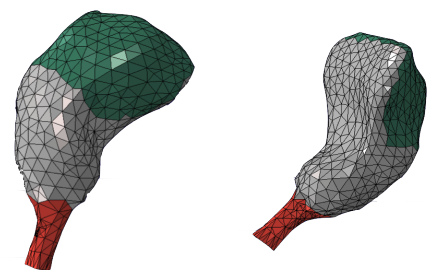

Fig. 13. Forces fields applied on uterus: (green) UC, (gray) UC, abdominal and diaphragm forces.

\section{H. Summary}

Fig. 14 presents our complete model with the different boundary conditions used for the organs.

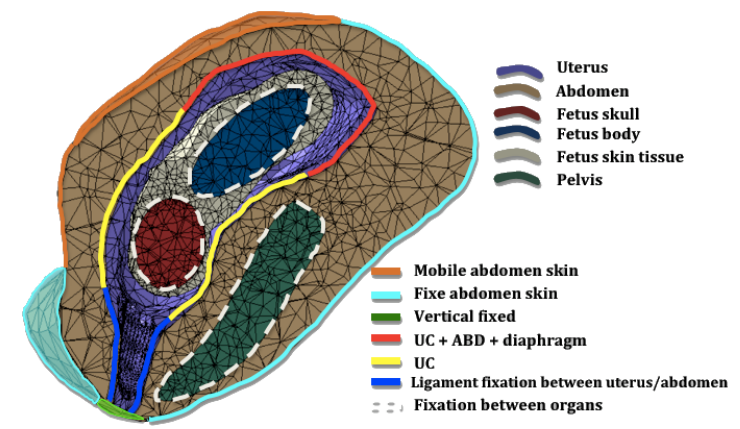

Fig. 14. Our biomechanical model of the female reproductive system in interaction with the fetus. Abbreviations: $A B D$ : abdominal forces; $U C$ : uterine contractions.

\section{RESUlts}

As we can't compare our results with images captured during a real childbirth, due to medical ethics restrictions, the validation is a difficult process. However, our medical partners have defined some verification parameters (uterus size, behavior of the bony pelvis with the sacrum movement, etc.) to validate our model.

\section{A. General behavior of the Simulation}

To simulate our biomechanical model, we used Abaqus FE software developed by Dassault Systems. With our simulation, the second stage of labor has a duration of 32 minutes (with an average velocity of $0.09 \mathrm{~mm} / \mathrm{s}$ ) which is similar to reality, with an average duration of 30 to 45 minutes. The execution time is 45 minutes on a Intel PC Core duo, 2.4GHz, 4Go RAM. Fig. 15 and 16 show some images of our 3D simulation. We have also included in this article a supplementary color MPEG file which contains several views of the $3 \mathrm{D}$ simulation for a better illustration of our 3D simulation results.
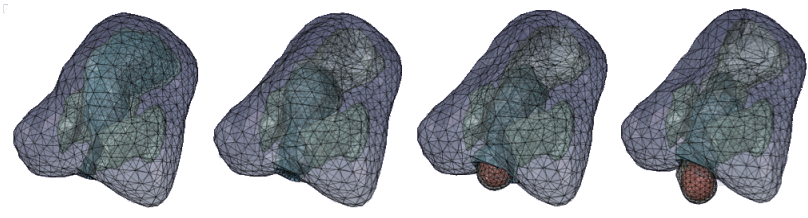

Fig. 15. Different phases of the 3D simulation of delivery.

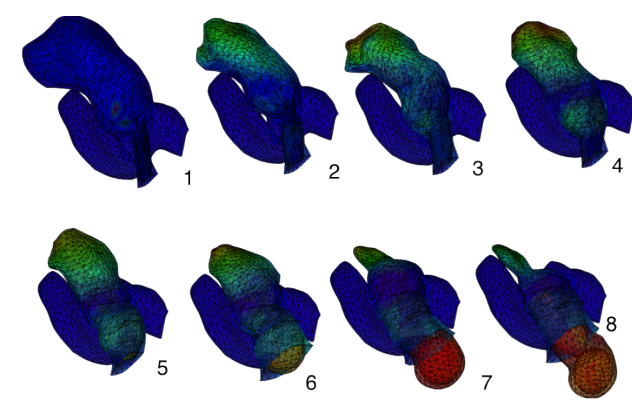

Fig. 16. Different phases of the 3D simulation of delivery.

\section{B. Kinematic Parameters}

The previous works in this area approximate the uterus and abdomen efforts by kinematic boundary constraints of the fetal head. In our work, the kinematic behavior of the fetal skull is computed according to exerted efforts. Fig. 17 shows the 3D evolution of the fetus head. We can see that it is essentially displaced in the coronal/sagittal axis. Moreover, during the simulation fetal head displacement amplitudes are $51.5 \mathrm{~mm}$ along the coronal/transversal axis, $63.5 \mathrm{~mm}$ along the sagittal/transversal axis and $184 \mathrm{~mm}$ along the coronal/sagittal axis. If we focus on the behavior in the coronal/sagittal axis, we can see that the fetal head velocity is not linear. Indeed, the parturient woman does not push continuously, involving increase or decrease in fetal head velocity. We can see this behavior in Fig. 18 which presents the evolution of the velocity of the fetal head along the sagittal/coronal axis. The negative parts of this curve indicate the inverse movement of the head.

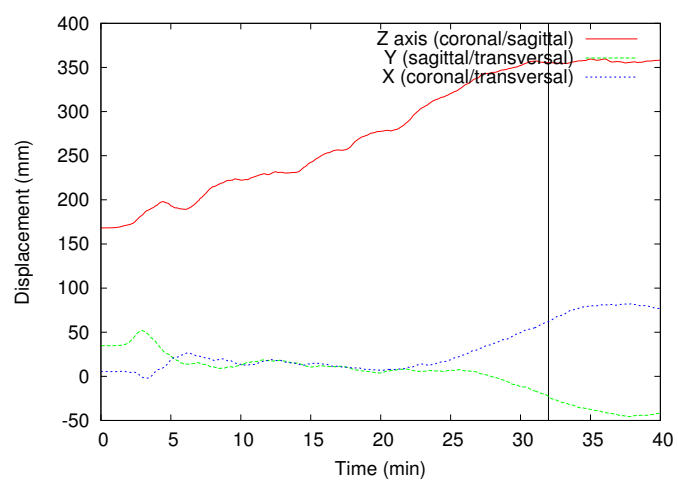

Fig. 17. Displacement of the fetal head along the coronal/transversal (X), sagittal/transversal (Y) and coronal/sagittal (Z) axis.

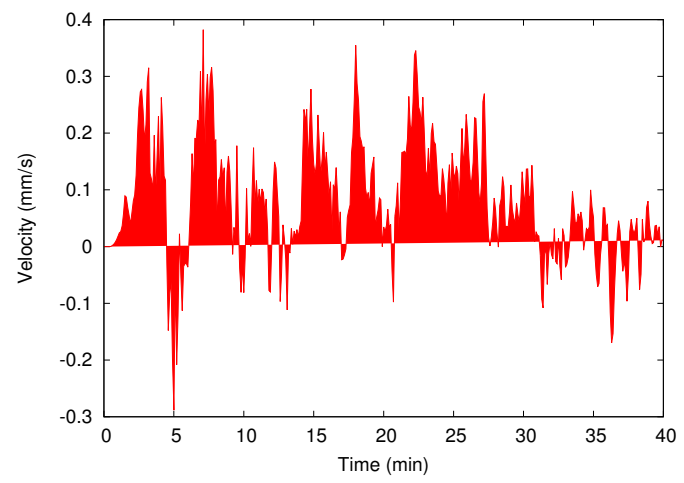

Fig. 18. Velocity of the fetal head along the coronal/sagittal (Z) axis. 


\section{Uterine behavior}

At the end of a real childbirth, the size of the uterus decreases by approximately $2 / 3$. This can be verified in our model by tracking the front-sagittal trajectory of a point at the top of the uterus over time and comparing it to a point on the lower part of the uterus. In Fig. 19, we can see that the distance between these two points is $230 \mathrm{~mm}$ at the beginning of the labor phase and $80 \mathrm{~mm}$ at the exit of the fetus. Consequently, we obtain the reduction of about $2 / 3$ in the uterine size, which is consistent with reality.

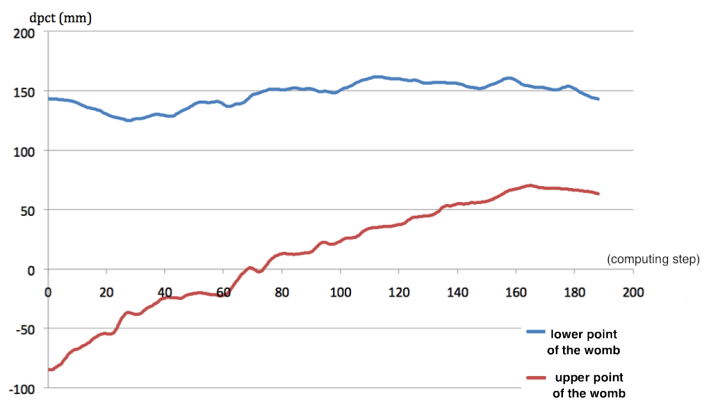

Fig. 19. Evolution of the trajectory of a front-sagittal point of the uterus.

Moreover, the uterine contractions experienced by the uterus during the second stage of labor involve the reduction in its volume during the descent of the fetus. To verify this behavior, let us consider two points of the uterus chosen in the transversal plane ( $c f$. Fig 20). Fig. 21 shows the movement of these two points during the simulation along the coronal/transversal axis. We can see that the displacements follow opposite directions. Consequently, uterine behavior corresponds to uterine contractions.

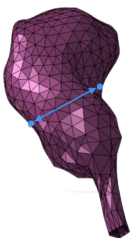

Fig. 20. The two points of the uterus followed during the simulation.

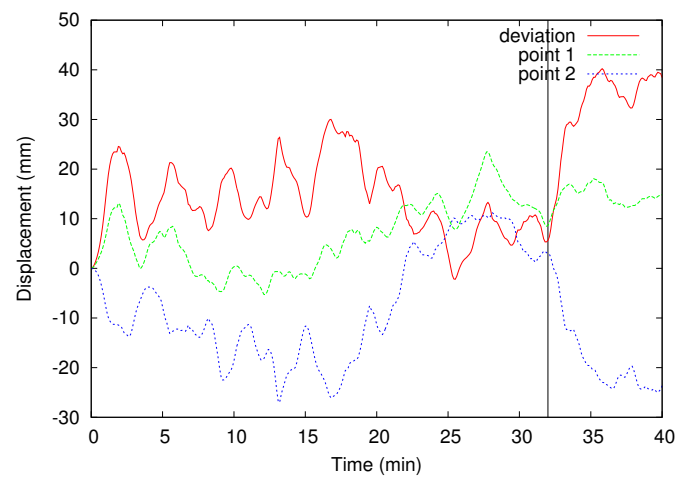

Fig. 21. Displacement of two points of the uterus during the simulation.

\section{Behavior of the Bony Pelvis}

Let us now consider the behavior of the bony pelvis. Fig. 22 shows its angular evolution in the sagittal plane. We can see two peaks in this curve. The first corresponds to the first contact of fetal head with the sacrum, which is pushed back by the bones of the fetal skull. Then, when the head enters the pelvic outlet, the second peak is caused by the passage of the rest of the body of the fetus. Moreover, as in reality, we can note that at the end of labor (32 minutes later), the pelvis does not return to its initial position.

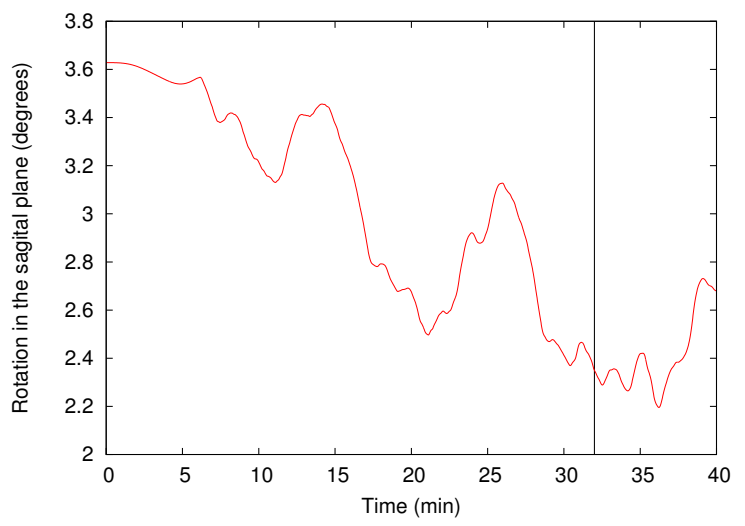

Fig. 22. Angular evolution of the pelvis in the sagittal plane.

\section{E. Behavior of the Fetal Head}

Fig. 23 shows the deformation of the head during the simulation. This deformation is caused by the compression of the skull by the pelvic muscles. Even if we did not model the head using a plastic law, our hyper-elastic model enables this deformation during the descent of the fetus enhancing the realism of the simulation.
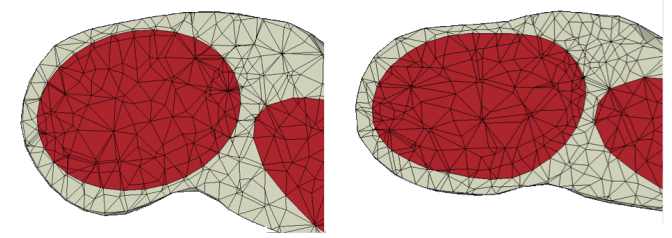

Fig. 23. Light crushing of the fetal head during the simulation.

\section{InTEGRATION IN A HAPTIC SIMULATOR}

We integrated our results (position of the fetal head according to time) in a physical simulator to reproduce our trajectory and compared it to the initial trajectory used in the simulator.

\section{A. Presentation of the Simulator}

The BirthSIM simulator [7], [8], [9] is composed of two sections ( $c f$. Fig. 24): (1) a mechanical section that consists of anthropomorphic models of the parturient pelvis and the fetal head and (2) an electro-pneumatic sections that reproduces the different efforts (uterine contractions, voluntary delivery efforts) and a rotary system controlled by a servomotor to position the fetal head in a given presentation. For the mechanical section, the silicon 3D model of the cranium of the fetus was manufactured by rapid prototyping techniques using CT-Scan data. 


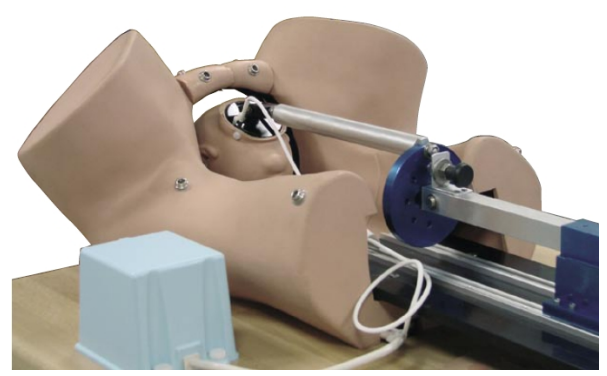

Fig. 24. The BirthSIM simulator composed of two sections: a mechanical section and an electro-pneumatic section.

This simulator includes several scenarios. We focused on one concerning a non-instrumented delivery. An entry signal (ES) is input to the system and then it is compared to a threshold value representing the resistance of the birth canal tissues $\left(F_{\text {resist }}\right)$. Initially this entry signal corresponds to the uterine contractions $\left(F_{U C}\right)$. These forces are represented by a Gaussian signal, which evolves regardless of interventions by the user. During training, the user can only control the abdominal forces $\left(F_{a b d}\right)$ by pressing a button at any time. These forces are then added to the uterine contractions involving an entry signal corresponding to the sum of the uterine contractions and the abdominal forces $\left(E S=F_{U C}+F_{a b d}\right)$. Then, only if the entry signal is higher than the threshold value, the fetal head moves in the birth canal ( $c f$. Fig 25).

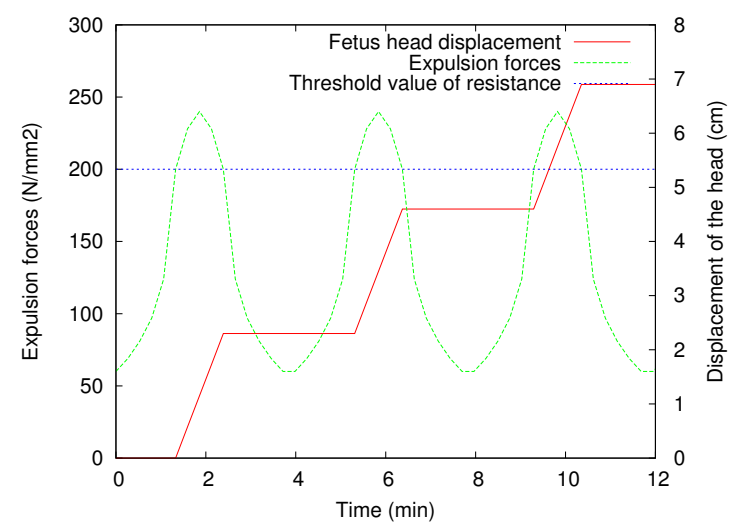

Fig. 25. Movement of the head induced by the expulsion forces.

\section{B. Integration of our Results}

By putting the position of the head of the fetus computed by the simulation in BirthSIM, the haptic device reproduces the descent of the head obtained by our simulation. To illustrate this result, we include in this paper a supplementary color MPEG file which shows both the biomechanical simulation and the use of its results in the BirthSIM simulator. Moreover, Fig. 26 shows the comparison of the BirthSIM trajectory of the fetal head used initially (in red) with the one obtained with our model (in green). To make this comparison, we chose the same initial position of the fetal head for both trajectories. As the BirthSIM model does not take morphology into account to increase or decrease the acceleration of the fetal head, the displacement of the fetus is supposed linear and cyclic (red curve). For this reason, we can see that the main difference between the two trajectories appears when the head leaves the pelvic floor (before 15 minutes). We can also see that both models converge at the same maximum amplitude $(15.5 \mathrm{~cm})$.

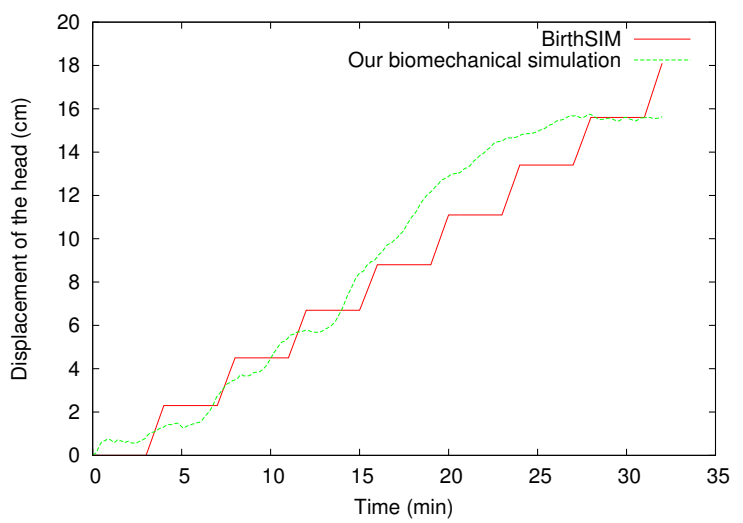

Fig. 26. Comparison between the BirthSIM trajectory of the fetal head with that obtained with our model.

Hence, our study still makes it possible to produce new trajectories that can be reproduced by BirthSIM, increasing its realism in the teaching of young obstetricians.

\section{Conclusion And Further Studies}

This paper presents a biomechanical modeling of interactions between the fetus and the parturient woman. Unlike all the existing Virtual Reality simulators that impose a pre-computed trajectory [19], [21], our biomechanical model allows a realistic simulation of the descent of the fetus through the birth canal during the second stage of labor. This simulation, taking into account the morphology of the organs, enables the computation of the real trajectory of the fetus.

To decrease the simulation computation time, we have only considered the main organs involved in childbirth: fetus, uterus, abdomen and pelvis. The geometrical model of the organs is produced from medical data (CT-scan and MRI) for women close to delivery. Moreover, the simulation is based on the principles of continuum mechanics using the finite element method.

The biomechanical model of the fetus is composed of three elements: the skin tissue, the body and the skull. The hyperelastic law of Neo-Hooke has been used to simulate the fetus, abdomen and uterus of the parturient woman, and the elastic law of Hooke has been used to simulate the pelvis. Then, the uterine contractions and expulsion forces (abdominal and diaphragm forces) were modeled as three force fields applied on different parts of the uterus, involving the descent of the fetus in the birth canal. Note that an additional force field has been added to simulate the constant pressure inside the body of the parturient woman.

Note that the validation is very difficult because we cannot compare our results with medical image acquisitions of a real childbirth, due to medical ethics restrictions. Consequently, we tried to verify the global behavior of the model by 
examining some characteristic features. The obstetricians of St Vincent de Paul Hospital, in Paris (Doctor G. Grangé and Professor C. Adamsbaum) have determined some validation features (behavior of the tip of the sacrum, decreasing of the uterus size, behavior of the bony pelvis, etc.). The quantitative comparisons with reality show that our model behaves quite well. Moreover, the trajectory computed during the simulation could improve an existing haptic device [7], [8] used for teaching young obstetricians.

Further studies concern the optimization or deterioration of the simulation to obtain interactive time simulation enabling it to be coupled to an haptic device. Moreover, several studies using our simulator for different pathological cases are planned. Our final aim is to obtain a real training system in collaboration with educational software specialists.

\section{ACKNOWLEDGMENT}

This work is partly financed by a grant from the GMCAO project of the ISLE cluster of the French Rhône-Alpes region. Special thanks to Jérémie Anquez (TELECOM ParisTech, CNRS, UMR5141, LTCI) for the segmentation of the medical data provided by Prof. Catherine Adamsbaum (St Vincent de Paul Hospital (AP-HP), Paris).

\section{REFERENCES}

[1] O. Dupuis, R. Silveira, T. Redarce, A. Dittmar, and R.-C. Rudigoz, "Operative vaginal delivery rate and neonatal associated complications in 2002 in the AURORE hospital network," Gynécologie Obstétrique et Fertilité, 2003.

[2] R. Moreau, M.-T. Pham, R. Silveira, T. Redarce, X. Brun, and O.Dupuis, "Design of a new instrumented forceps: Application to safe obstetrical forceps blade placement," IEEE Transactions on Biomedical Engineering, vol. 7, no. 54, july 2007.

[3] E. J. Kim, P. Theprungsirikul, M. K. McDonald, E. D. Gurewithsch, and R. H. Allen, "A biofidelic birthing simulator," IEEE Engineering in Medicine and Biology Magazine, vol. 24, no. 6, pp. 34-39, 2005.

[4] R. H. Allen, "On the mechanical aspects of shoulder dystocia and birth injury," Clinical obstetrics and gynecology, vol. 50(3), p. 607623, 2007.

[5] J. S. Eggert, M. S. Eggert, and P. Vallejo, "Interactive education system for teaching patient care. patent no. us2003/0081968a1," May 2003. [Online]. Available: http://www.freepatentsonline.com/y2003/0091968.html

[6] X. Li, J. A. Kruger, P. Nash, and M. Nielsen, "Modeling childbirth: elucidating the mechanisms of labor," Wiley Interdisciplinary Reviews: Systems Biology and Medicine, vol. 2, no. 4, pp. 460-470, aug 2010.

[7] R. Silveira, M.-T. Pham, T. Redarce, M. Btemps, and O. Dupuis, "A new mechanical birth simulator: BirthSIM," in IEEE/RSJ International Conference on Intelligent Robots and Systems (IROS'04), Sendai, Japan, 2004, pp. 3948-3954.

[8] R. Moreau, "Le simulateur BirthSIM : un outil complet pour la formation sans risque en obstétrique," Ph.D. dissertation, Institut National des Sciences Appliquées, Lyon, France, 2007.

[9] O. Dupuis, A. Dittmar, G. Delhomme, T. Redarce, M. Betemps, and R. Silveira, "Simulateur fonctionnel et anatomique daccouchement," aug 2003, French Licence. Licence Number: 0309569.

[10] R.-J. Muffler, "AV-8B HARRIER II training capabilities," in AIAA Flight Simulator Technologies Conference, St Louis, MO, USA, 1985, pp. 1115.

[11] D. Aulignac, C. Laugier, J. Troccaz, and S. Vieira, "Towards a realistic echographic simulator," Medical Image Analysis, vol. 10, pp. 71-81, 2006.

[12] H. K. Cakmak, "Advanced Surgical Training in Laparoscopy with VEST Simulators," in 2eme Worshop on Basic Anatomy and advanced Technology in Laparoscopic Surgery, Kiel Allemagne, 2003.

[13] S. Cotin, H. Delingette, J.-M. Clement, V. Tasseti, J. Marescaux, and N. Ayache, "Volumetric deformable models for simulation of laparoscopic surgery," in International Symposium on Computer and communication Systems for Image Guided Diagnosis and Therapy, Computer Assisted Radiology, Paris, France, 1996.
[14] P. Dubois, J.-F. Rouland, P. Meseure, S. Karpf, and C. Chaillou, "Simulator for laser photocoagulation in ophtalmology," IEEE Transaction in Biomedical Engineering, vol. 42, no. 7, 1995.

[15] P.-Y. Zambelli, C. Bregand, S. Dewarrat, G. Marti, C. Baur, and P. Leyvraz, "Planning and navigation solution in resurfacing hips surgery: a way to reduce the surgical approach," in Poster session, 3rd Annual meeting of the International Society Orthopaedic Surgery, Marbella, Spain, 2003.

[16] R. Gardner, "Simulation and simulator technology in obstetrics: past, present and future," Expert Review in Obstetrics \& Gynecology, vol. 2, no. 6, pp. 775-90, nov 2007.

[17] R. Gardner and D. B. Raemer, "Simulation in obstetrics and gynecology," Obstetrics and gynecology clinics of North America, vol. 35, no. 1, pp. 97-127, mar 2008.

[18] G. Letterie, "How virtual reality may enhance training in obstetrics and gynecology," American journal of obstetrics and gynecology, vol. 187, pp. S37-S40, sep 2002.

[19] J.-D. Boissonnat and B. Geiger, "3D simulation of delivery," in Visualization 93, G. M. Nielson and D. Bergeron, Eds. San Jose CA: IEEE Computer Society Press, 1993, pp. 416-419.

[20] B. Geiger, "Three-dimensional modeling of human organs and its application to diagnosis and surgical planning," Ph.D. dissertation, Ecole des Mines de Paris, 1993.

[21] A. Kheddar, C. Devine, M. Brunel, C. Duriez, and O. Sidony, "Preliminary design of a childbirth simulator haptic feedback," in IEEE/RSJ, International Conference on Inteligent Robots and Systems, vol. 4, 2004, pp. 3270-3275.

[22] T. Obst, R. Burghart, E. Ruckhberle, and R. Reiner, "The delivery simulator: A new application of medical VT," in MMVR 2004, Newport Beach, 2004, pp. 281-287.

[23] R. J. Lapeer and R. W. Prager, "Fetal head moulding: finite element analysis of a fetal skull subjected to uterine pressures during the first stage of labour,' Journal of Biomechanics, vol. 34, pp. 1125-1133, 2001.

[24] R. J. Lapeer, M. Chen, and J. Villagrana, "An Augmented Reality based Simulation of Obstetric Forceps Delivery," in Third IEEE and ACM International Symposium on Mixed and Augmented Reality (ISMAR 2004), nov 2004, pp. 274-275.

[25] R. J. Lapeer, "A mechanical contact model for the simulation of obstetric forceps delivery in a virtual/augmented environment," Studies in Health Technology and Informatics, vol. 111, pp. 284-289, feb 2005.

[26] J. Martins, M. Pato, E. Pires, R. Natal-Jorge, M. Paraente, and T. Mascarenhas, "Finite element studies of the deformation of the pelvic floor," Ann N Y Academy of Scineces, vol. doi:10.1196/annals.1389.19, pp. 316334, 2007.

[27] D. Riethmuller, P. Roth, A. Martin, R. Maillet, and J.-P. Schaal, "Benefits of ultrasonography in the delivery room," Gynécologie, Obstétrique et fertilité, 2004.

[28] X. Li, J. Kruger, M. Nash, and P. Nielsen, "Anisotropic effects of the levator ani muscle during childbirth," Biomechanics and modeling in mechanobiology, aug 2010.

[29] J. Mizrahi and Z. Karni, "A mechanical model for uterine muscle activity durng labor and delivery," Israel Journal of Technology, vol. 13, pp. $185-191,1975$

[30] J. Mizrahi, Z. Karni, and W. Polishuk, "Isotropy and anisotropy of uterine muscle during labor contraction," Journal of Biomechanics, vol. 13 , no. 3, pp. 211-218, 1980.

[31] R. Buttin, F. Zara, B. Shariat, and T. Redarce, "A biomechanical model of the female reproductive system and the fetus for the realization of a childbirth virtual simulator," in IEEE Engineering in Medicine and Biology Society (EMBC'09), sep 2009.

[32] E. Mazza, A. Nava, M. Bauer, R. Winter, M. Bajka, and G. Holzapfel, "Mechanical properties of the human uterine cervix: an in vivo study," Medical Image Analysis, vol. 10, no. 2, pp. 125-136, sep 2006.

[33] Y. Fung, Biomechanics. Mechanical properties of living tissues, 2nd ed. Springer, 1993.

[34] M. Bauer, E. Mazza, M. Jabareen, L. Sultan, M. Bajka, U. Lang, R. Zimmermann, and G. Holzapfel, "Assessment of the in vivo biomechanical properties of the human uterine cervix in pregnancy using the aspiration test: A feasibility study," European Journal of Obstetrics \& Gynecology and Reproductive Biology, vol. 144, pp. S77-S81, 52009.

[35] M. Dufour and M. Pillu, Biomécanique fonctionnelle : Membres-TêteTronc. Masson, 2007.

[36] M. Attene and B. Falcidieno, "ReMESH: An interactive environment to edit and repair triangle meshes," in Shape Modeling and Applications (SMI), 2006, pp. 271-276.

[37] H. yamada, Strength of biological materials. Williams \& Wilkins in Baltimore, 1970. 
[38] M. Dalstra, R. Huiskes, A. Odgaard, and L. Van Erning, "Mechanical and textural properties of pelvic trabecular bone," Journal of biomechanics, vol. 26, no. 4-5, pp. 523-535, 1993.

[39] J.-P. Schaal, D. Riethmuller, R. Maillet, and M. Uzan, Mécanique et Technique Obstétricales, 3rd ed. sauramps medical, fev 2007.

[40] L. Dubrisay and C. Jeannin, Précis d'accouchement. Lamarre, 1946.

[41] P. Kamina, Anatomie clinique: anatomie générale, membre, 3rd ed. Maloine, 2006. 